\title{
El derecho a la salud sexual y reproductiva en la Argentina: un análisis a partir de la variación de la mortalidad por enfermedades de transmisión sexual entre los quinquenios 1997-2001 y 2009-2013
}

\author{
Eleonora Soledad Rojas Cabrera ${ }^{1}$
}

Recibido: 6/01/2017

Aceptado: 10/03/2017

\section{Resumen}

Este trabajo tiene por objeto examinar si el comportamiento de la mortalidad por enfermedades de transmisión sexual (ETS) en la Argentina conlleva avances hacia el ejercicio universal del derecho a la salud sexual y reproductiva, e identificar los desafíos que supondrá cumplir con los compromisos asumidos en el ámbito internacional. Para ello se calculan y analizan las tasas de mortalidad por sexo, edad y causa en los quinquenios 1997-2001 y 2009-2013.

Los resultados revelan logros relativos a la reducción de la mortalidad por ETS y, particularmente, por el virus de la inmunodeficiencia humana $(\mathrm{VIH}) /$ síndrome de inmunodeficiencia adquirida (SIDA), la sífilis y la hepatitis B. Además, muestran un descenso de la mortalidad femenina asociada al virus del papiloma humano (VPH). Se observa también, en las edades más avanzadas, un aumento de la mortalidad por el

\footnotetext{
Técnico en Investigación Socioeconómica, Especialista en Diseño de Encuestas y Análisis de Datos para la Planificación Social, y Magíster y Doctora en Demografía. Investigadora Asistente del Centro de Investigaciones y Estudios sobre Cultura y Sociedad (CIECS) del Consejo Nacional de Investigaciones Científicas y Técnicas (CONICET) y la Universidad Nacional de Córdoba (UNC). Correo electrónico: eleonorarojascabrera@gmail.com.
} 
VIH/SIDA y de la mortalidad masculina vinculada al VPH. Ello evidencia la necesidad de reforzar las medidas implementadas desde una perspectiva integral.

Palabras clave: derechos humanos, salud sexual y reproductiva, enfermedades de transmisión sexual, mortalidad, Argentina.

\section{Abstract}

This study examines whether the behaviour of mortality caused by sexually transmitted diseases (STDs) in Argentina shows progress in the exercise of the universal right to sexual and reproductive health care, and to identify the challenges implicit in the commitments undertaken at the international level. To this effect, mortality rates by sex, age and cause have been calculated and analysed for the 1997-2001 and 2009-2013 five-year periods.

The results show achievements in reducing STD mortality and especially in mortality linked to human immunodeficiency virus (HIV)/acquired immunodeficiency syndrome (AIDS), syphilis and hepatitis B. A drop in female mortality associated with human papilloma virus (HPV) was also observed, as was an increase in HIV/AIDS mortality in older segments of the population and in male mortality linked to HPV. This highlights the need to reinforce the implementation of comprehensive health care measures.

Key words: human rights, sexual and reproductive health, sexually transmitted diseases, mortality, Argentina.

\section{Résumé}

Cette étude a pour but d'analyser si le comportement de la mortalité due à des maladies sexuellement transmissibles (MST) en Argentine évolue vers l'exercice universel du droit à la santé sexuelle et reproductive, ainsi que de préciser les défis qui découleront du respect des engagements contractés à l'échelle internationale. Pour ce faire, nous avons calculé et analysé les taux de mortalité par sexe, âge et cause au cours des quinquennats 1997-2001 et 2009-2013.

Les résultats font apparaître des progrès en matière de réduction de la mortalité associée aux MST et, en particulier, au virus de l'immunodéficience humaine $(\mathrm{VIH}) /$ syndrome d'immunodéficience acquise (sida), à la syphilis et à l'hépatite $B$. Ils révèlent également une baisse de la mortalité féminine associée au virus du papillome humain (VPH). À un âge plus avancé, on observe également une augmentation de la mortalité liée au VIH/sida, ainsi que de la mortalité masculine associée au VPH. Toutes ces observations suggèrent qu'il est nécessaire de renforcer les mesures appliquées d'un point de vue intégral.

Mots clés: droits humains, santé sexuelle et reproductive, maladies sexuellement transmissibles, mortalité, Argentine. 


\section{Introducción}

Las enfermedades de transmisión sexual (ETS) comprometen el ejercicio del derecho a la salud sexual y reproductiva de las personas, al limitar el disfrute de la vida sexual sin riesgos y, en ocasiones, la capacidad de procrear. Además, repercuten negativamente en el ejercicio efectivo de los restantes derechos, convirtiendo a quienes las contraen en víctimas de estigmatización y discriminación social.

En razón de lo anterior y de la alta y creciente incidencia de las ETS en el mundo, la comunidad internacional instó a los Estados a reducir la morbimortalidad por este grupo de causas para 2015, con énfasis en el virus de la inmunodeficiencia humana (VIH)/ síndrome de inmunodeficiencia adquirida (SIDA), dado que la situación global se agrava con su aparición. Así se expresa en el Programa de Acción de la Conferencia Internacional sobre la Población y el Desarrollo (CIPD) de 1994 (párr. 7.29), en que se establecen medidas para cumplir con este propósito. Dichas medidas están orientadas a: i) la prevención, diagnóstico y tratamiento de las ETS (párr. 7.30); ii) la capacitación de los recursos humanos involucrados (párr. 7.31); iii) la difusión de información y la educación de la población para promover el despliegue de una conducta sexual responsable y la prevención eficaz de dichas enfermedades (párr. 7.32); iv) el suministro de medios confiables (preservativos de buena calidad) en favor de la prevención (párr. 7.33), y v) el fomento de investigaciones para erradicar su propagación (párr. 8.29)².

Una vez finalizado el plazo para el logro de los objetivos del Programa de Acción de la CIPD, y dada la persistencia de limitaciones para reducir la morbimortalidad por ETS en algunos grupos específicos de la población, los países acordaron redoblar los esfuerzos para alcanzar, en 2030, la salud sexual y reproductiva de manera universal. Así quedó establecido, globalmente, en los Objetivos de Desarrollo Sostenible (ODS) (Objetivo 3) y, en el ámbito regional, en el Consenso de Montevideo sobre Población y Desarrollo, aprobado en 2013 (medida prioritaria 38).

La República Argentina adhirió a la CIPD y a los compromisos relativos a las metas a ser alcanzadas en 2030. Es así como ha desplegado, desde la década de 1990, acciones para reducir la morbimortalidad por ETS en todo el territorio nacional. Sin embargo, adaptó formalmente la normativa a los compromisos asumidos recién en 2003 , cuando se creó el Programa Nacional de Salud Sexual y Procreación Responsable (Ley núm. 25.673), que consta de objetivos concretos para abordar integralmente la problemática en un marco caracterizado por la provisión de información y de servicios orientados a toda la población 3 .

\footnotetext{
2 Posteriormente, en otros planes de acción basados en los enunciados de la CIPD se retoman y reafirman sus intenciones. Entre ellos, pueden mencionarse la Plataforma de Acción de la Cuarta Conferencia Mundial sobre la Mujer de 1995 (objetivo estratégico C.3) y los Objetivos de Desarrollo del Milenio (ODM) (meta 6.A).

3 Dicho Programa se complementa con el Programa Nacional de Educación Sexual Integral (Ley núm. 26.150). Creado en 2006, este Programa tiene por objeto transmitir en las escuelas conocimientos acerca del tema en favor de la prevención de las ETS, la concientización y la lucha contra la discriminación hacia quienes padecen infecciones de transmisión sexual.
} 
En este contexto, cabe preguntarse: ¿el comportamiento de la mortalidad por ETS en la Argentina durante los últimos años ha posibilitado avances en el ejercicio del derecho a la salud sexual y reproductiva a nivel nacional4? ¿Varían las conclusiones si el análisis de la mortalidad por ETS se realiza de acuerdo al sexo, la edad y la causa específica de muerte de las personas? ¿Qué desafíos se evidencian para el cumplimiento de los compromisos en el futuro?

A fin de dar respuesta a estos interrogantes se calculan y analizan las tasas de mortalidad específicas correspondientes a los quinquenios 1997-2001 y 2009-2013, sobre la base de información proveniente de la Dirección de Estadísticas e Información de Salud (DEIS) y del Instituto Nacional de Estadística y Censos (INDEC) 5 .

\section{A. Datos y métodos}

Como se anticipara en la introducción, en este trabajo se utilizan datos procedentes de la DEIS y del INDEC. Los primeros consisten en estadísticas de nacimientos y defunciones, y en la desagregación de estas últimas según sexo, edad y causa de mortalidad asociada a ETS $^{6}$. Los datos del INDEC son proyecciones de población desagregadas por sexo y grupos quinquenales de edad?

Se consideran las siguientes causas de mortalidad incluidas en la Clasificación Estadística Internacional de Enfermedades y Problemas Relacionados con la Salud, Décima Revisión (CIE-10): i) las infecciones cuyo modo de transmisión es predominantemente sexual, es decir, la sífilis (A50-A53), la infección gonocócica (A54), la clamidiasis (A55-A56), el chancro blando (A57), el granuloma inguinal (A58), la tricomoniasis (A59), el herpes genital (A60), otras ETS (A63) y ETS no especificadas (A64); ii) la hepatitis aguda tipo B (B16); iii) la enfermedad por el VIH (B20-B24), y iv) los tumores malignos relacionados con el virus del papiloma humano (VPH) ( $\mathrm{C}_{10}, \mathrm{C}_{21}, \mathrm{C}_{51}, \mathrm{C}_{52}, \mathrm{C}_{53}$ y $\mathrm{C} 60$, códigos asociados, respectivamente, a los tumores de orofaringe, ano, vulva, vagina, cuello uterino y pene).

Cabe agregar que, aun cuando existen distintas formas de transmisión del VIH, dada la imposibilidad de desagregar las muertes de acuerdo a dichas formas de transmisión se considera que todas ellas deben su origen a la práctica de la actividad sexual sin preservativo

$4 \quad$ Si bien en este artículo la atención está puesta en el análisis de la mortalidad, no dejan de reconocerse los esfuerzos progresivos del Estado argentino para avanzar en el registro de la morbilidad asociada a las causas de interés. Estos esfuerzos se sintetizan, por ejemplo, en el Sistema Nacional de Vigilancia Epidemiológica (SINAVE) (MSAL, 2007).

5 En este trabajo se aborda la mortalidad por infecciones de transmisión sexual y por otras causas no consideradas infecciones en sí mismas. Por lo tanto, se utiliza la denominación ETS para referirse a las causas estudiadas a lo largo del texto.

6 Merece una mención especial la calidad de las estadísticas vitales del país. Al respecto, según información difundida por el Centro Latinoamericano y Caribeño de Demografía (CELADE)-División de Población de la Comisión Económica para América Latina y el Caribe (CEPAL), la diferencia relativa entre el número de nacimientos registrados en las estadísticas vitales y los estimados en las proyecciones de población disminuyó del 3,3\% al 1,6\% entre los quinquenios 1995-2000 y 2005-2010, mientras que la diferencia correspondiente al número de defunciones se redujo del 2,6\% al 1,6\% entre los mismos quinquenios (CELADE, 2015).

$7 \quad$ Los totales de población y de muertes por ETS se presentan en el cuadro A1.1 del anexo incluido al final de este artículo. 
con una persona que vive con el virus (salvo aquellas que ocurren en los primeros años de vida, asociadas generalmente al contagio a través de la madre $)^{8}$. Este supuesto se apoya en datos publicados por la Dirección de SIDA y Enfermedades de Transmisión Sexual (DSyETS), según los cuales el $90 \%$ de las personas diagnosticadas en el país en el trienio 2012-2014 contrajeron el VIH mediante la vía referida (DSyETS, 2015).

Por otro lado, si bien solo una proporción de los tumores mencionados es atribuible al VPH, se decide considerar la totalidad de las muertes ligadas a ellos debido a las limitaciones propias de la información empleada, que no permiten distinguir entre las muertes vinculadas al VPH y las no vinculadas a este virus. Esta decisión encuentra sustento en información difundida por el Instituto Nacional del Cáncer de los Estados Unidos, según la cual prácticamente todos los casos de cáncer de cuello uterino son causados por infecciones asociadas al VPH, en tanto que la proporción de casos ligados a esta patología desciende al $95 \%$, el $70 \%$, el $65 \%$, el $50 \%$ y el $35 \%$ en los cánceres de ano, orofaringe, vagina, vulva y pene, respectivamente (Instituto Nacional del Cáncer de los Estados Unidos, 2015).

A partir de los datos descritos se calcularon las tasas de mortalidad específicas correspondientes a los quinquenios 1997-2001 y 2009-2013 según sexo, grupo de edad y causa de la defunción. Dichas tasas resultan del cociente entre el promedio de defunciones de cada quinquenio y la población proyectada a mediados de cada uno de los períodos considerados (esto es, en 1999 y en 2011 , respectivamente).

En relación con el cálculo de las tasas de mortalidad se realizan algunas aclaraciones. En primer lugar, la elección de los quinquenios responde al interés por estudiar el comportamiento de la mortalidad por ETS antes y después de la creación del Programa Nacional de Salud Sexual y Procreación Responsable, hecho a partir del cual la Argentina adaptó la normativa a los compromisos asumidos en el plano internacional. En el mismo sentido, dado que las ETS son clasificadas sobre la base de la CIE-10, se establece como punto de partida de la presente investigación 1997 (año en que dicha Clasificación comenzó a aplicarse en el país) y como cierre 2013 (último año del cual existía información disponible en el momento de la realización de este estudio).

En segundo lugar, se contemplan los grupos de edad sugeridos por la Organización Panamericana de la Salud (OPS) para el cálculo de las tasas de mortalidad por enfermedades transmisibles: niños menores de 1 año, personas de 1 a 14 años, de 15 a 24 años, de 25 a 44 años, de 45 a 64 años y de 65 años y más (OPS, 2014).

En tercer lugar, en el cálculo de la tasa de mortalidad infantil (es decir, la relativa a los niños menores de 1 año), el denominador es el total de los nacidos vivos registrados a mediados de cada uno de los quinquenios seleccionados (OPS, 2014).

Por último, todas las tasas de mortalidad se expresan en números por cada 100.000 habitantes (o nacidos vivos, según el caso), de acuerdo con la unidad de medida empleada en los estudios de mortalidad por grupos de causas o causas específicas (OPS, 2014).

$8 \quad$ Entre las distintas formas de transmisión del VIH se incluyen: i) las relaciones sexuales sin protección con una persona que vive con el VIH; ii) la transfusión de sangre contaminada; iii) el uso de agujas, jeringas u otros objetos punzocortantes contaminados, y iv) la transmisión de madre a hijo durante el embarazo, el parto o el período de lactancia. 


\section{B. Resultados y discusión}

\section{La mortalidad por ETS en la Argentina según sexo, grupo de edad y causa específica}

La tasa de mortalidad por ETS es superior entre las mujeres. Dicha tasa ha tendido a reducirse en el período estudiado con independencia del sexo, aunque lo ha hecho con mayor énfasis entre los varones. Concretamente, entre los quinquenios investigados la tasa de mortalidad masculina pasó de 7,6 a 6,0 por cada 100.000 varones, mientras que la femenina descendió de 8,2 a 7,9 por cada 100.000 mujeres.

Los valores presentados podrían ser considerados bajos en algún sentido. Sin embargo, resultan significativos en el marco de los derechos humanos, dado que se refieren a muertes susceptibles de ser evitadas. Además, como se comentara en la introducción de este artículo, el padecimiento de ETS compromete seriamente la salud sexual y reproductiva de las personas afectadas y las torna vulnerables a sufrir situaciones de discriminación en diferentes contextos.

Al considerar la edad se observa que la tasa de mortalidad masculina alcanza su máximo nivel en el grupo de 25 a 44 años a inicios del período estudiado y en el grupo de 45 a 64 años a fines de dicho período. Este desplazamiento del punto máximo de la tasa de mortalidad guarda relación con su descenso en la población menor de 45 años (fundamentalmente entre los niños menores de 1 año y los adultos de 25 a 44 años) y con su aumento a partir de esa edad (véase el cuadro 1).

Cuadro 1

Argentina: tasas de mortalidad asociada a enfermedades de transmisión sexual, por sexo y grupo de edad, 1997-2001 y 2009-2013

(Por cada 100.000 habitantes o nacidos vivos)

\begin{tabular}{lcccccc}
\hline & \multicolumn{7}{c}{ Grupo de edad } \\
\cline { 2 - 7 } & $\begin{array}{c}\text { Menores } \\
\text { de 1 año }\end{array}$ & $\begin{array}{c}\text { De 1 a } \\
14 \text { años }\end{array}$ & $\begin{array}{c}\text { De 15 a } \\
\text { 24 años }\end{array}$ & $\begin{array}{c}\text { De 25 a } \\
\text { 44 años }\end{array}$ & $\begin{array}{c}\text { De 45 a } \\
64 \text { años }\end{array}$ & $\begin{array}{c}\text { 6n años } \\
\text { y más }\end{array}$ \\
\hline $1997-2001$ & 8,1 & 0,5 & 2,2 & 18,7 & 7,2 & 7,8 \\
$2009-2013$ & 3,5 & 0,2 & 1,0 & 9,2 & 11,7 & 8,7 \\
\hline $1997-2001$ & 8,3 & 0,4 & 1,6 & 11,4 & 14,2 & 18,4 \\
$2009-2013$ & 3,7 & 0,1 & 0,8 & 9,6 & 15,0 & 16,7 \\
\hline
\end{tabular}

Fuente: Elaboración propia, sobre la base de información de la Dirección de Estadísticas e Información de Salud (DEIS), Ministerio de Salud de la Nación.

La situación de las mujeres es similar a la de los varones hasta los 24 años. Luego, la tasa de mortalidad femenina aumenta de manera gradual. Este patrón se mantiene en el quinquenio 2009-2013, pero se observan diferencias según el grupo de edad, al reducirse 
la tasa de mortalidad de todos los grupos contemplados, salvo del grupo de 45 a 64 años (véase el cuadro 1).

La desagregación de las muertes por causas específicas permite observar un predominio de aquellas atribuidas al VIH/SIDA y a los tumores relacionados con la presencia del VPH (en ese orden, en lo que respecta a los varones, y a la inversa, en lo que se refiere a las mujeres) (véase el cuadro 2).

Cuadro 2

\section{Argentina: tasas de mortalidad asociadas a las principales enfermedades de transmisión sexual por sexo, grupo de edad y causa, 1997-2001 y 2009-2013}

(Por cada 100.000 habitantes o nacidos vivos)

\begin{tabular}{|c|c|c|c|c|c|c|c|c|c|c|c|c|}
\hline & \multicolumn{12}{|c|}{ Grupo de edad } \\
\hline & \multicolumn{2}{|c|}{$\begin{array}{l}\text { Menores } \\
\text { de } 1 \text { año }\end{array}$} & \multicolumn{2}{|c|}{$\begin{array}{c}\text { De } 1 \text { a } \\
14 \text { años }\end{array}$} & \multicolumn{2}{|c|}{$\begin{array}{l}\text { De } 15 \text { a } \\
24 \text { años }\end{array}$} & \multicolumn{2}{|c|}{$\begin{array}{l}\text { De } 25 \text { a } \\
44 \text { años }\end{array}$} & \multicolumn{2}{|c|}{$\begin{array}{l}\text { De } 45 \text { a } \\
64 \text { años }\end{array}$} & \multicolumn{2}{|c|}{$\begin{array}{l}65 \text { años } \\
\text { y más }\end{array}$} \\
\hline & $\begin{array}{l}1997- \\
2001\end{array}$ & $\begin{array}{l}2009- \\
2013\end{array}$ & $\begin{array}{l}1997- \\
2001\end{array}$ & $\begin{array}{l}2009- \\
2013\end{array}$ & $\begin{array}{l}1997- \\
2001\end{array}$ & $\begin{array}{l}2009- \\
2013\end{array}$ & $\begin{array}{l}1997- \\
2001\end{array}$ & $\begin{array}{l}2009- \\
2013\end{array}$ & $\begin{array}{l}1997- \\
2001\end{array}$ & $\begin{array}{l}2009- \\
2013\end{array}$ & $\begin{array}{l}1997- \\
2001\end{array}$ & $\begin{array}{l}2009- \\
2013\end{array}$ \\
\hline \multicolumn{13}{|c|}{ Varones } \\
\hline Sífilis & 4,3 & 2,9 & - & - & - & - & - & - & 0,1 & - & 0,2 & 0,1 \\
\hline Hepatitis B & 0,1 & - & - & - & - & - & 0,1 & - & 0,4 & 0,2 & 1,0 & 0,2 \\
\hline $\begin{array}{l}\text { Virus de la } \\
\text { inmunodeficiencia } \\
\text { humana }(\mathrm{VIH})\end{array}$ & 3,8 & 0,7 & 0,5 & 0,2 & 2,1 & 0,9 & 18,4 & 9,0 & 5,3 & 9,4 & 1,5 & 2,6 \\
\hline $\begin{array}{l}\text { Tumores asociados } \\
\text { al virus del papiloma } \\
\text { humano (VPH) }\end{array}$ & - & - & - & - & - & - & 0,2 & 0,2 & 1,5 & 2,0 & 5,1 & 5,9 \\
\hline \multicolumn{13}{|c|}{ Mujeres } \\
\hline Sífilis & 4,0 & 2,6 & - & - & - & - & - & - & - & - & 0,1 & - \\
\hline Hepatitis B & 0,1 & - & - & - & - & - & - & - & 0,2 & 0,1 & 0,5 & 0,1 \\
\hline $\begin{array}{l}\text { Virus de la } \\
\text { inmunodeficiencia } \\
\text { humana }(\mathrm{VIH})\end{array}$ & 4,2 & 1,0 & 0,4 & 0,1 & 1,3 & 0,6 & 5,6 & 4,4 & 1,2 & 2,7 & 0,3 & 0,7 \\
\hline $\begin{array}{l}\text { Tumores asociados } \\
\text { al virus del papiloma } \\
\text { humano (VPH) }\end{array}$ & - & - & - & - & 0,2 & 0,2 & 5,8 & 5,2 & 12,7 & 12,2 & 17,4 & 15,8 \\
\hline
\end{tabular}

Fuente: Elaboración propia, sobre la base de información de la Dirección de Estadísticas e Información de Salud (DEIS), Ministerio de Salud de la Nación.

Nota: Se excluyen las tasas de mortalidad asociadas a las restantes enfermedades de transmisión sexual por presentar valores inferiores a los exhibidos en el cuadro.

Más allá de esta lectura, la variación de la mortalidad por el VIH/SIDA da cuenta de la labor del país en favor de su reducción, sobre todo en lo que se refiere a los varones. La tasa de mortalidad por el VIH/SIDA de este grupo de población cayó de 6,6 a 4,9 por cada 100.000 varones entre los dos quinquenios analizados, mientras que la participación relativa de las muertes vinculadas a esta causa en el total de las muertes por ETS relevadas varió del $86,3 \%$ al $81,5 \%$ en el mismo período.

Como contrapartida, la tasa de mortalidad femenina por el VIH/SIDA, si bien presentó valores inferiores en términos comparativos, prácticamente no registró cambios entre los quinquenios investigados (la tasa de mortalidad fue de 2,1 y 2,0 por cada 100.000 mujeres en 
los quinquenios 1997-2001 y 2009-2013, respectivamente, y la participación relativa del 25,9\% y el $24,9 \%$, respectivamente).

La reducción de la tasa de mortalidad por el VIH/SIDA guarda relación con la evolución del número de casos notificados a consecuencia de las acciones realizadas por el Estado para disminuir la incidencia de la enfermedad 9 . En tal sentido, a nivel nacional, entre 2004 y 2013 la tasa de diagnósticos de personas con el VIH descendió de 22,7 a 18,8 por cada 100.000 varones, y de 14,1 a 9,0 por cada 100.000 mujeres (DSyETS, 2015) ${ }^{10}$.

Esta evolución diferencial de la tasa de diagnósticos por sexo derivó, a su vez, en un incremento de la razón entre el número de casos notificados de varones y el número de casos notificados de mujeres (que pasó de 1,3 a 1,7 entre los trienios 2006-2008 y 2012-2014) (DSyETS, 2015).

Por otro lado, el comportamiento de la tasa de mortalidad también se asocia al volumen creciente de personas que viven con el VIH que reciben habitualmente tratamiento para contrarrestar los efectos de la patología. Al respecto, gracias a la implementación de la Ley Nacional de SIDA núm. 23.798 de 1990 (que garantiza la atención y el tratamiento en hospitales públicos y sin costo adicional para obras sociales y prepagas), más de 69.000 personas reciben actualmente medicamentos antirretrovirales, mientras que 7 de cada 10 lo hacen en el ámbito del sistema sanitario estatal (Argentina, Congreso de la Nación, 1990; DSyETS, 2015).

Al considerarse la edad, se observa que el comportamiento de la tasa de mortalidad por el VIH/SIDA es similar en la población de o a 24 años con independencia del sexo. Aunque en el quinquenio 1997-2001 los valores más elevados se registraron en los grupos etarios de 25 a 44 años (ambos sexos), de 45 a 64 años (solo en el caso de los varones) y de menores de 1 año (ambos sexos), en ese orden, posteriormente los valores se redujeron en todos los grupos de edad comprendidos entre o y 44 años (sobre todo en el grupo de varones de 25 a 44 años) y se incrementaron en la población de 45 años y más (véase el cuadro 2).

El descenso de la tasa de mortalidad en las poblaciones más jóvenes está en consonancia con el comportamiento de la incidencia del VIH en cada tramo abordado. En efecto, disminuyó la proporción de casos positivos por transmisión perinatal ${ }^{11}$, mientras que aumentó la tasa de diagnósticos del VIH entre los varones de 20 a 24 años y se redujo entre aquellos de 30 a 39 años y entre las mujeres de 25 a 39 años ${ }^{12}$.

En el otro extremo, el incremento de la tasa de mortalidad en las edades más avanzadas podría ser explicado tanto por el uso progresivo de antirretrovirales por parte de las personas que viven con el VIH (con el consecuente incremento de su esperanza de vida), como por

9 En la Argentina, la notificación de estos casos es obligatoria desde 1990 (art. 10, Ley Nacional de SIDA núm. 23.798).

10 La tasa de diagnósticos del VIH surge del cociente entre el número de diagnósticos del VIH registrados durante un año en un lugar determinado y la población de ese lugar a mediados de ese año.

11 Esta cuestión se refleja en otros indicadores, como el porcentaje de embarazadas de 15 a 24 años que viven con el VIH con respecto al total de las embarazadas de ese tramo de edad que se realizaron las pruebas de detección del VIH, el cual se redujo del 0,64\% al 0,36\% entre 2000 y 2006 (CNCPS/PNUD, 2012).

12 Entre los bienios 2004-2005 y 2012-2013, la tasa de diagnósticos del VIH varió de 21,6 a 31,9, de 60 a 40 y de 35 a 20 por cada 100.000 habitantes, respectivamente, en los tres grupos poblacionales mencionados (DSyETS, 2015). 
el momento en que se produce el diagnóstico de los nuevos casos (que es fundamental para protegerse, evitar contagios y recibir oportunamente tratamiento).

Esto último configura un reto en materia de políticas públicas, ya que si bien la DSyETS dispone de abastecimiento para más de 1,25 millones de determinaciones de carga viral para tamizaje del VIH, distribuido en todas las provincias del país, se observa que a partir de los 15 años aumenta la proporción de diagnósticos tardíos en ambos sexos, destacándose el grupo de 55 a 64 años, en el que los diagnósticos tardíos superan a los oportunos (DSyETS, 2015). De manera excepcional, dicha proporción desciende ligeramente a partir de los 65 años, probablemente en concordancia con la disminución de la tasa de mortalidad femenina vinculada (DSyETS, 2015).

Por su parte, si bien los tumores relacionados con el VPH lideran la estructura de la mortalidad femenina asociada a las ETS (la tasa de mortalidad fue cercana a 6 por cada 100.000 en ambos quinquenios, y la participación relativa fue del $71,4 \%$ y del 73,8\%, respectivamente), llama la atención el incremento de la tasa de mortalidad masculina más allá de su valor (en los quinquenios considerados la tasa de mortalidad fue de o,7 y de 1,0 por cada 100.000 , y la participación relativa fue del $9,4 \%$ y del $16,1 \%$, respectivamente), sobre todo a partir de los 45 años (véase el cuadro 2).

En particular, entre los varones las tasas de mortalidad asociadas a estos tumores crecen fundamentalmente en el caso de los de pene y en mayor medida entre la población de 65 años y más (véase el cuadro 3).

Cuadro 3

Argentina: tasas de mortalidad asociadas a tumores relacionados con el virus del papiloma humano, por sexo, grupo de edad y localización del tumor, 1997-2001 y 2009-2013

(Por cada 100.000 habitantes)

\begin{tabular}{|c|c|c|c|c|c|c|}
\hline \multirow{3}{*}{ Localización } & \multicolumn{6}{|c|}{ Grupo de edad } \\
\hline & \multicolumn{2}{|c|}{ De 25 a 44 años } & \multicolumn{2}{|c|}{ De 45 a 64 años } & \multicolumn{2}{|c|}{65 años y más } \\
\hline & $1997-2001$ & $2009-2013$ & $1997-2001$ & 2009-2013 & $1997-2001$ & $2009-2013$ \\
\hline \multicolumn{7}{|c|}{ Varones } \\
\hline Orofaringe & - & - & 0,4 & 0,6 & 0,7 & 1,0 \\
\hline Ano & - & 0,1 & 0,2 & 0,4 & 0,9 & 0,9 \\
\hline Pene & 0,1 & 0,1 & 0,8 & 1,1 & 3,5 & 4,0 \\
\hline \multicolumn{7}{|c|}{ Mujeres } \\
\hline Orofaringe & - & - & 0,1 & 0,1 & 0,1 & 0,2 \\
\hline Ano & - & - & 0,1 & 0,4 & 0,8 & 0,8 \\
\hline Vulva & 0,1 & - & 0,4 & 0,5 & 4,7 & 3,8 \\
\hline Vagina & - & - & 0,2 & 0,2 & 1,0 & 1,1 \\
\hline Cuello uterino & 5,6 & 5,2 & 11,8 & 11,0 & 10,8 & 10,0 \\
\hline
\end{tabular}

Fuente: Elaboración propia, sobre la base de información de la Dirección de Estadísticas e Información de Salud (DEIS), Ministerio de Salud de la Nación.

Nota: Se excluye a la población menor de 25 años pues las tasas de mortalidad son iguales o inferiores a 0,2 por cada 100.000 habitantes en todos los casos. 
En lo que respecta a las mujeres, aunque las muertes debidas a tumores de cuello uterino y de vulva, en ese orden, son las más habituales (especialmente desde los 25 años, alcanzándose las tasas máximas en el grupo de 45 a 64 años en el primer caso, y en el de 65 años y más en el segundo), las tasas de mortalidad descendieron en el período considerado (véase el cuadro 3). Esta reducción podría estar ligada al incremento sostenido de la proporción de mujeres de 25 a 65 años que acceden al examen de Papanicolau con cierta regularidad, como un medio para diagnosticar y tratar oportunamente la enfermedad (INDEC/MSAL, 2014a).

No puede concluirse lo mismo respecto de las muertes ligadas a los tumores de vagina, ano y orofaringe, cuyas tasas se incrementan, principalmente desde los 45 años (véase el cuadro 3).

Seguidamente se destacan la hepatitis B y la sífilis (se trata de la tercera y la cuarta causa de mortalidad por ETS, respectivamente), aunque estas están ligadas a tasas de mortalidad inferiores a 0,2 por cada 100.000 habitantes o nacidos vivos, en general, y tienden a descender en ambos sexos (véase el cuadro 2).

En tanto que la reducción de la tasa de mortalidad por la primera de estas causas (que es superior entre los varones y afecta principalmente a las poblaciones adultas) podría estar asociada a la introducción de la vacuna contra la hepatitis B en el calendario oficial de vacunación ${ }^{13}$, la disminución de la mortalidad debida a la segunda causa mencionada (que se manifiesta mayormente entre los niños y las niñas que no han alcanzado 1 año de edad) podría relacionarse con la creciente proporción de mujeres embarazadas que acceden a control prenatal14 (UNICEF/Ministerio de Desarrollo Social, 2013).

\section{Algunos desafíos vinculados a la reducción de la morbimortalidad por ETS}

A partir de los resultados exhibidos, cabe reflexionar acerca de los desafíos que se vislumbran en el país en relación con los niveles de mortalidad asociados a determinadas causas y grupos poblacionales, ya sea para mantener su reducción, o bien para revertir su tendencia al aumento.

En primera instancia, cabe mencionar los desafíos relacionados con el acceso a la información para la prevención de las ETS. Existen numerosas acciones orientadas a la difusión de conocimientos para prevenir las ETS, que se llevan a cabo tanto en el ámbito

13 La vacuna se aplica en las primeras 12 horas de vida (dosis neonatal) y posteriormente adquiere carácter universal, debiendo iniciar o completar el esquema (de tres dosis en total) todas las personas a partir de los 15 años de edad (MSAL, 2007).

14 En la Argentina, según datos de la Encuesta sobre Condiciones de Vida de Niñez y Adolescencia (ECOVNA) aplicada a nivel nacional en el bienio 2011-2012, el $98 \%$ de las mujeres de 15 a 49 años que dieron a luz en los dos años anteriores al relevamiento se realizaron, al menos, un control prenatal; a su vez, el 89,8\% de ellas asistieron a cuatro controles o más (UNICEF/Ministerio de Desarrollo Social, 2013). Estos porcentajes están en consonancia con la proporción de partos atendidos por personal especializado, que aumentó del $96,9 \%$ en 1990 al $99,4 \%$ en 2010 (CNCPS/PNUD, 2012). 
público como en el privado. Entre las primeras se incluye la labor de la DSyETS, en el seno de la cual se publican contenidos sobre el tema en distintas redes sociales, se dictan talleres virtuales, se distribuyen folletos específicos y se realizan periódicamente campañas informativas ("Elegí saber", por ejemplo, tiene por objeto fomentar el test de diagnóstico del VIH, mientras que otras acciones se orientan a la promoción del uso correcto del preservativo) (DSyETS, 2015).

Entre las segundas, figura la labor de algunas organizaciones no gubernamentales. A modo de ejemplo cabe mencionar a la Fundación Huésped, entre cuyas acciones se incluye la realización, cada año, de un programa unitario de ficción con actores de reconocida trayectoria, que se transmite por un canal abierto de televisión cada 1 de diciembre con motivo del Día Mundial del SIDA (Fundación Huésped, s/f).

Sin embargo, pareciera que estos esfuerzos resultan aún insuficientes, ya que el nivel de conocimientos de la población sobre las ETS difiere según la causa específica de la enfermedad y el sector social de pertenencia de las personas. Es así como, aunque los resultados de la Encuesta sobre Condiciones de Vida de Niñez y Adolescencia (ECOVNA) 2011-2012 y de la Encuesta Nacional sobre Salud Sexual y Reproductiva (ENSSyR) 2013 señalan que más del $95 \%$ de la población ha escuchado alguna información acerca de la existencia del VIH/SIDA (porcentaje que aumenta a medida que se incrementa el nivel de instrucción de las personas encuestadas), ese valor disminuye considerablemente cuando se indaga acerca del conocimiento de las principales formas de prevenir su transmisión (UNICEF/Ministerio de Desarrollo Social, 2013; INDEC/MSAL, 2014c).

Esta realidad se torna más grave si se tienen en cuenta otras ETS. Al respecto, según los resultados de la ENSSyR 2013 solo el 35\% de los varones y el 65\% de las mujeres manifestaron haber oído hablar acerca del VPH. Más alarmante aún es el hecho de que dichos valores son inferiores cuando se refieren a las poblaciones más jóvenes o cuando las preguntas se refieren a conocimientos más específicos (tales como los relacionados con la asociación entre el virus y el cáncer de cuello uterino), y casi siempre son menores los porcentajes correspondientes a los varones (INDEC/MSAL, 2014b).

Si bien estos porcentajes mejoran en lo que respecta a la hepatitis B y la sífilis (y lo hacen en mayor medida según aumentan la edad y el nivel de instrucción), no puede concluirse lo mismo respecto de las restantes ETS. Un caso extremo se refleja en los porcentajes de población que escuchó hablar alguna vez acerca de la leucorrea y la tricomoniasis, que no superan el $15 \%$, con independencia del sexo y la edad de los encuestados (INDEC/MSAL, 2014C).

Otros desafíos se vinculan con el acceso a métodos de prevención y protección, y con su utilización ${ }^{15}$. Más allá de que la DSyETS promueve activamente el empleo del preservativo y otros métodos de barrera, y de que cuenta con una política para su distribución gratuita,

15 Recién en 2011 se introdujo la vacuna contra el VPH en el calendario oficial de inmunización para las niñas de 11 años con el objetivo de prevenir la aparición del cáncer de cuello uterino (PRONACEI/MSAL, 2014). En consecuencia, no es posible identificar aún avances ni desafíos en tal sentido. 
la cantidad de preservativos y otros dispositivos entregados no siempre permanece estable o aumenta con el tiempo ${ }^{16}$. Peor aún, según datos de la ENSSyR 2013, solo el 22,2\% de los varones de entre 14 y 59 años y el 32,2\% de las mujeres de entre 14 y 49 años acceden a dichos métodos por esta vía (INDEC/MSAL, 2014c).

También resulta alarmante que la práctica del uso del preservativo no esté todavía del todo instalada en la sociedad. Por una parte, si bien diversos estudios permiten observar un incremento paulatino de la utilización de este método desde la primera relación sexual y en las relaciones posteriores (Binstock y Gogna, 2015; Binstock y Pantelides, 2015; Rojas Cabrera, Moyano y Peláez, 2015), esta práctica no resulta del todo adecuada ni sistemática (Binstock y Pantelides, 2015) ${ }^{17}$.

Por otra parte, según los resultados de la ENSSyR 2013 dicha práctica decrece con la edad (INDEC/MSAL, 2014c), hecho que permitiría explicar el incremento de la tasa de mortalidad por el VIH/SIDA en los grupos de población de edades más avanzadas.

Asimismo, existen asimetrías intersectoriales en función de diversos factores. Entre ellos, se cuentan los años de educación formal (el método es más utilizado a medida que aumentan estos últimos, por lo que el riesgo de contraer ETS se incrementa en las poblaciones socioeconómicamente más desfavorecidas), el tipo de unión (el preservativo es más utilizado en aquellas de tipo ocasional) y el sexo (en el plano de las relaciones íntimas, los varones suelen influir negativamente en la utilización de cualquier método de prevención) (Schufer y Geldstein, 2005).

También contribuyen a estas diferencias el nivel de conocimiento acerca de las ventajas del preservativo como método de doble protección (este método es más empleado como herramienta de planificación familiar, sobre todo en las parejas estables) y, como se mencionara antes, el nivel de conocimiento de las vías de contagio de las ETS (que trae aparejadas conductas de riesgo, tales como la práctica de sexo anal sin preservativo, mayormente entre personas del mismo sexo) (Pantelides y Manzelli, 2005; Schufer y Geldstein, 2005).

Otra cuestión a tener en cuenta se relaciona con las acciones vinculadas al diagnóstico oportuno de los casos. En tal sentido, se destaca el hecho de que algunas medidas de control se focalizan exclusivamente sobre ciertos sectores de la población. Entre ellas, cabe mencionar la realización progresiva de la prueba del VIH a las mujeres durante el embarazo ${ }^{18}$ y las acciones orientadas a fomentar la realización de la prueba de Papanicolau

16 A modo de ejemplo, puede mencionarse que el número promedio de preservativos entregados mensualmente por la DSyETS varió entre 2006 y 2015, registrándose años de gran distribución (por ejemplo, 3.715.774 en 2010 y 4.161.294 en 2013), seguidos de otros en que la distribución alcanzó valores comparativamente bajos (por ejemplo, 2.486.823 en 2011 y 2.231 .088 en 2014) (DSyETS, 2015).

17 Además de los riesgos de contraer ETS, esta cuestión trae aparejadas otras consecuencias, entre ellas, los embarazos no planificados que tienen lugar en la adolescencia.

18 Aunque los datos de la ECOVNA 2011-2012 permiten observar que el 72\% del total de las mujeres que tuvieron un hijo o hija en los dos años anteriores a la Encuesta accedieron a la prueba del VIH durante el control prenatal, también revelan desafíos por superar, sobre todo en materia de información. En efecto, del total de mujeres a las que se practicó la prueba, solo el $32 \%$ obtuvieron asesoramiento respecto de la enfermedad, en tanto que poco más del $70 \%$ recibieron los resultados de dicho test (UNICEF/Ministerio de Desarrollo Social, 2013). 
para prevenir el cáncer de cuello uterino o proceder a su tratamiento de manera adecuada y a tiempo.

Por el contrario, no se han desplegado hasta el momento acciones dirigidas a promover la realización de controles para reducir el número de muertes atribuidas a causas como el cáncer de pene, ni tampoco de otras zonas corporales que, en ocasiones, también intervienen en las relaciones sexuales (ano y orofaringe) ${ }^{19}$.

Todos estos desafíos sugieren la necesidad de repensar el diseño y la ejecución de algunas acciones llevadas a cabo desde la esfera pública, así como de redoblar los esfuerzos realizados en determinados sentidos. Entre ellos, se incluyen los relacionados con los contenidos relativos a la salud sexual y reproductiva que se difunden en las escuelas en el marco de la aplicación de la Ley núm. 26.150 (Programa Nacional de Educación Sexual Integral) y con el establecimiento del momento oportuno para su difusión.

Esta última reflexión se fundamenta en los resultados de un estudio realizado por Binstock y Gogna (2015), cuyas principales conclusiones señalan que en ciertas ocasiones los conocimientos son impartidos con posterioridad a la primera relación sexual, y que los jóvenes y adolescentes que los reciben no siempre los califican como útiles o muy útiles.

\section{Conclusiones}

La información presentada en este trabajo permite identificar progresos en lo que se refiere a la reducción de la mortalidad por ETS en la Argentina desde la adaptación de la normativa nacional a los compromisos asumidos en el plano internacional. Estos logros se observan en el descenso que registra la tasa de mortalidad por ETS en general y, particularmente, las tasas de mortalidad asociadas al VIH (en las poblaciones más jóvenes), la sífilis (fundamentalmente en el grupo etario de los niños) y la hepatitis B (sobre todo en la población de 65 años y más).

Además, los datos presentados permiten observar un descenso de la mortalidad femenina por tumores ligados al VPH, debido principalmente a la disminución de la tasa de mortalidad asociada a los tumores de cuello de útero.

Por otro lado, se observa, en las edades más avanzadas, un aumento de la tasa de mortalidad por el VIH, que podría atribuirse al uso de antirretrovirales que inciden en la extensión de la esperanza de vida de las personas afectadas, pero que también podría estar vinculado al diagnóstico tardío de la enfermedad. Se observa asimismo un incremento de la mortalidad masculina a causa de los tumores asociados al VPH (de pene, ano y orofaringe).

19 Esta lectura cobra especial relevancia en el caso de las relaciones sexuales con personas del mismo sexo, fundamentalmente entre los varones. Así lo corroboran datos publicados por la DSyETS, según los cuales, entre 2012 y 2014, los porcentajes de varones diagnosticados con el VIH se distribuyeron equitativamente entre quienes se contagiaron a través de una relación sexual con una mujer y quienes lo hicieron como resultado de una relación sexual con otro varón, a diferencia de las mujeres, que en su mayoría se contagiaron mediante una relación sexual con un varón (DSyETS, 2015). 
Esto último deja en evidencia la necesidad de redoblar los esfuerzos en relación con las medidas implementadas desde una perspectiva integral. Entre ellas, se cuentan las relacionadas con el acceso a la información desde edades tempranas y con la promoción del diagnóstico oportuno y de los controles pertinentes con independencia del sexo y la edad de las personas. Más allá de que las tasas de mortalidad asociadas a cada causa específica puedan ser consideradas bajas en algún sentido, sus implicaciones en el contexto de los derechos humanos hacen necesaria la profundización de dichas acciones.

\section{Bibliografía}

Argentina, Congreso de la Nación (2006), Ley núm. 26.150. Programa Nacional de Educación Sexual Integral, Buenos Aires, octubre.

(2003), Ley núm. 25.673. Programa Nacional de Salud Sexualy Procreación Responsable, Buenos Aires, mayo. _ (1990), Ley Nacional de SIDA núm. 23.798, Buenos Aires, septiembre.

Binstock, G. y M. Gogna (2015), "La iniciación sexual entre mujeres de sectores vulnerables en cuatro provincias argentinas", Sexualidad, Saludy Sociedad, $\mathrm{N}^{\circ} 20$, Río de Janeiro, Centro Latinoamericano de Sexualidad y Derechos Humanos.

Binstock, G. y E. Pantelides (2015), "Las conductas sexuales y reproductivas de los adolescentes: la iniciación sexual. Resultados de la ENSSyR 2013", documento presentado en la conferencia XIII Jornadas Argentinas de Estudios de Población, Salta, Asociación de Estudios de Población de la Argentina (AEPA), 16 a 18 de septiembre.

CELADE (Centro Latinoamericano y Caribeño de Demografía-División de Población de la CEPAL) (2015), "Evaluaciones e indicadores de cobertura y calidad: experiencias regionales", documento presentado en el Taller sobre los Principios y Recomendaciones para un Sistema de Estadísticas Vitales, Revisión 3, para Países de América del Sur, Santiago, 9 a 12 de noviembre.

CEPAL (Comisión Económica para América Latina y el Caribe) (2013), Consenso de Montevideo sobre Población y Desarrollo (LC/L.3697), Santiago [en línea] http://repositorio.cepal.org/bitstream/ handle/11362/21835/S20131037_es.pdf? sequence $=4$.

CNCPS/PNUD (Consejo Nacional de Coordinación de Políticas Sociales/Programa de las Naciones Unidas para el Desarrollo) (2012), Objetivos de Desarrollo del Milenio. Informe País Argentina 2012, Buenos Aires, diciembre.

DSyETS (Dirección de SIDA y Enfermedades de Transmisión Sexual) (2015), Boletín sobre el VIH-SIDA e ITS en la Argentina, $\mathrm{N}^{\circ} 32$, Buenos Aires, diciembre.

Fundación Huésped (s/f), "Programas de TV", Buenos Aires [en línea] http://www.huesped.org.ar/ proyectos/programas-de-tv/.

INDEC/MSAL (Instituto Nacional de Estadísticas y Censos/Ministerio de Salud de la Nación) (2014a), $3^{\circ}$ Encuesta Nacional de Factores de Riesgo. Para Enfermedades no Transmisibles. Presentación de los principales resultados, Buenos Aires.

_(2014b), ENSSyR. Encuesta Nacional sobre Salud Sexual y Reproductiva 2013. Documento para la utilización de las bases de datos usuario, Buenos Aires.

_-(2014c), Encuesta Nacional sobre Salud Sexual y Reproductiva 2013. ENSSyR 2013. Presentación de resultados, Buenos Aires. 
Instituto Nacional del Cáncer de los Estados Unidos (2015), "Virus del papiloma humano y el cáncer" [en línea] http://www.cancer.gov/espanol/cancer/causas-prevencion/riesgo/germenesinfecciosos/hoja-informativa-vph.

MSAL (Ministerio de Salud de la Nación) (2007), Manual de normas y procedimientos de vigilancia y control de enfermedades de notificación obligatoria. Revisión nacional 2007, Buenos Aires.

Naciones Unidas (2015), "Proyecto de documento final de la cumbre de las Naciones Unidas para la aprobación de la agenda para el desarrollo después de 2015" (A/69/L.85), agosto.

_(2000), "Declaración del Milenio" (A/RES/55/2), septiembre.

_(1996), Informe de la Cuarta Conferencia Mundial sobre la Mujer, Beijing, 4 a 15 de septiembre de 1995 (A/CONF.177/20/Rev.1), Nueva York.

_ (1995), Informe de la Conferencia Internacional sobre la Población y el Desarrollo, El Cairo, 5 a 13 de septiembre de 1994 (A/CONF.171/13/Rev.1), Nueva York.

OPS (Organización Panamericana de la Salud) (2014), "Glosario de Indicadores Básicos de la OPS", Washington, D.C., septiembre.

OPS/OMS (Organización Panamericana de la Salud/Organización Mundial de la Salud) (1995), Clasificación Estadística internacional de enfermedades y problemas relacionados con la salud. Décima revisión, Washington, D.C.

Pantelides, E. y H. Manzelli (2005), La salud sexual y reproductiva: también un asunto de hombres, Buenos Aires, Organización Panamericana de la Salud (OPS).

PRONACEI/MSAL (Programa Nacional de Control de Enfermedades Inmunoprevenibles/Ministerio de Salud de la Nación) (2014), Vacuna contra el virus del papiloma humano(VPH). Lineamientos técnicos. Transición a vacuna cuadrivalente. Manual del vacunador, Buenos Aires.

Rojas Cabrera, E., S. Moyano y E. Peláez (2015), "Progresos y desafíos en el ejercicio del derecho a la salud sexual y reproductiva en la Argentina de los últimos 40 años. Una aproximación a partir de información relativa a "la primera vez", documento presentado en las XIII Jornadas Argentinas de Estudios de Población, Salta, Asociación de Estudios de Población de la Argentina (AEPA), 16 a 18 de septiembre.

Schufer, M. y R. Geldstein (2005), "Los varones jóvenes de Buenos Aires y el sexo seguro", VII Jornadas Argentinas de Estudios de Población, Buenos Aires, Universidad Nacional de Tucumán (UNT)/ Asociación de Estudios de Población de la Argentina (AEPA).

UNICEF/Ministerio de Desarrollo Social (Fondo de las Naciones Unidas para la Infancia/Ministerio de Desarrollo Social) (2013), Encuesta sobre Condiciones de Vida de Niñez y Adolescencia, 2011-2012. Principales resultados, Buenos Aires. 


\section{Anexo A1}

Cuadro A1.1

Argentina: población y total de muertes por enfermedades de transmisión sexual por sexo y grupo de edad, 1997-2001 y 2009-2013

(En números de personas)

\begin{tabular}{|c|c|c|c|c|c|c|}
\hline & \multicolumn{6}{|c|}{ Grupo de edad } \\
\hline & $\begin{array}{l}\text { Menores } \\
\text { de } 1 \text { año }\end{array}$ & $\begin{array}{c}\text { De } 1 \text { a } \\
14 \text { años }\end{array}$ & $\begin{array}{l}\text { De } 15 \text { a } \\
24 \text { años }\end{array}$ & $\begin{array}{l}\text { De } 25 \text { a } \\
44 \text { años }\end{array}$ & $\begin{array}{l}\text { De } 45 \text { a } \\
64 \text { años }\end{array}$ & $\begin{array}{l}65 \text { años } \\
\text { y más }\end{array}$ \\
\hline & \multicolumn{6}{|c|}{ Varones } \\
\hline Población, 1999 & 351820 & 4840780 & 3315400 & 4796900 & 3187500 & 1448500 \\
\hline $\begin{array}{l}\text { Total de muertes por } \\
\text { enfermedades de } \\
\text { transmisión sexual, } \\
\text { 1997-2001 }\end{array}$ & 143 & 115 & 358 & 4476 & 1153 & 568 \\
\hline Población, 2011 & 391094 & 4773260 & 3461172 & 5873289 & 3802683 & 1733283 \\
\hline \multirow[t]{2}{*}{$\begin{array}{l}\text { Total de muertes por } \\
\text { enfermedades de } \\
\text { transmisión sexual, } \\
2009-2013\end{array}$} & 69 & 44 & 169 & 2713 & 2220 & 757 \\
\hline & \multicolumn{6}{|c|}{ Mujeres } \\
\hline Población, 1999 & 331277 & 4697423 & 3243900 & 4842200 & 3443500 & 2079500 \\
\hline $\begin{array}{l}\text { Total de muertes por } \\
\text { enfermedades de } \\
\text { transmisión sexual, } \\
\text { 1997-2001 }\end{array}$ & 137 & 91 & 262 & 2768 & 2438 & 1909 \\
\hline Población, 2011 & 366591 & 4622823 & 3361178 & 5852411 & 4123057 & 2539655 \\
\hline $\begin{array}{l}\text { Total de muertes por } \\
\text { enfermedades de } \\
\text { transmisión sexual, } \\
2009-2013\end{array}$ & 67 & 27 & 127 & 2819 & 3101 & 2119 \\
\hline
\end{tabular}

Fuente: Elaboración propia, sobre la base de información de la Dirección de Estadísticas e Información de Salud (DEIS), Ministerio de Salud de la Nación. 